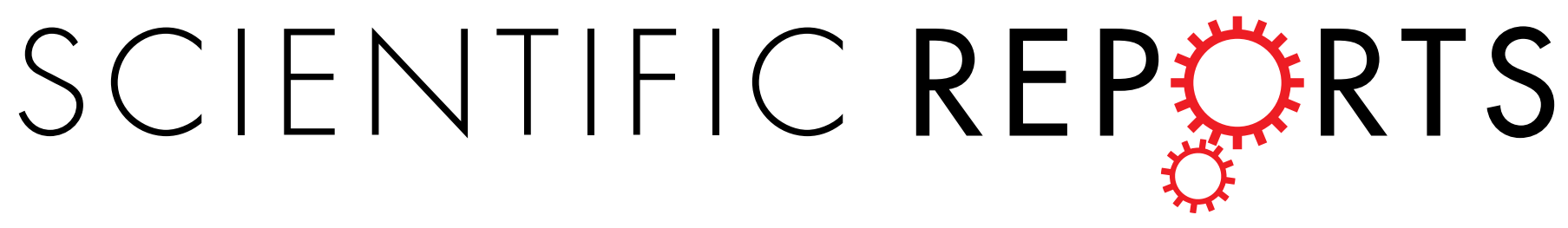

\title{
OPEN Erratum: Spring maize yield, soil water use and water use efficiency under plastic film and straw mulches in the Loess Plateau
}

Wen Lin, Wenzhao Liu \& Qingwu Xue

Scientific Reports 6:38995; doi: 10.1038/srep38995; published online 15 December 2016; updated on 15 March 2017

This article had a row omitted in Table 1, the correct table is below:

\begin{tabular}{|l|c|c|c|c|c|c|c|}
\hline & & \multicolumn{3}{|c|}{$\mathbf{0 - 6 0 0} \mathbf{c m}$} & \multicolumn{3}{c|}{ 0-300 cm } \\
\cline { 3 - 8 } Treatment & Precipitation mm & CK & SM & PM & CK & SM & PM \\
\hline 2009 & 357 & $-27 \mathrm{ab}$ & $-55 \mathrm{a}$ & $1 \mathrm{~b}$ & $-26 \mathrm{ab}$ & $-50 \mathrm{a}$ & $-2 \mathrm{~b}$ \\
\hline 2010 & 543 & $-144 \mathrm{~b}$ & $-177 \mathrm{a}$ & $-142 \mathrm{~b}$ & $-143 \mathrm{a}$ & $-143 \mathrm{a}$ & $-151 \mathrm{a}$ \\
\hline 2011 & 468 & $-107 \mathrm{a}$ & $-115 \mathrm{a}$ & $-40 \mathrm{~b}$ & $-103 \mathrm{a}$ & $-126 \mathrm{a}$ & $-47 \mathrm{~b}$ \\
\hline 2012 & 344 & $32 \mathrm{a}$ & $49 \mathrm{a}$ & $60 \mathrm{a}$ & $20 \mathrm{a}$ & $18 \mathrm{a}$ & $44 \mathrm{~b}$ \\
\hline 2013 & 400 & $-69 \mathrm{a}$ & $-35 \mathrm{a}$ & $-66 \mathrm{a}$ & $-78 \mathrm{a}$ & $-54 \mathrm{a}$ & $-70 \mathrm{a}$ \\
\hline 2014 & 268 & $89 \mathrm{a}$ & $96 \mathrm{a}$ & $141 \mathrm{a}$ & $111 \mathrm{a}$ & $111 \mathrm{a}$ & $164 \mathrm{~b}$ \\
\hline 2015 & 361 & $105 \mathrm{~b}$ & $113 \mathrm{~b}$ & $64 \mathrm{a}$ & $93 \mathrm{ab}$ & $109 \mathrm{~b}$ & $73 \mathrm{a}$ \\
\hline $7-\mathrm{yr}-$ change & - & $44 \mathrm{a}$ & $-7 \mathrm{a}$ & $-9 \mathrm{a}$ & $51 \mathrm{~b}$ & $22 \mathrm{ab}$ & $8 \mathrm{a}$ \\
\hline
\end{tabular}

Table 1.

(c) (i) This work is licensed under a Creative Commons Attribution 4.0 International License. The images or other third party material in this article are included in the article's Creative Commons license, unless indicated otherwise in the credit line; if the material is not included under the Creative Commons license, users will need to obtain permission from the license holder to reproduce the material. To view a copy of this license, visit http://creativecommons.org/licenses/by/4.0/

(C) The Author(s) 2017 